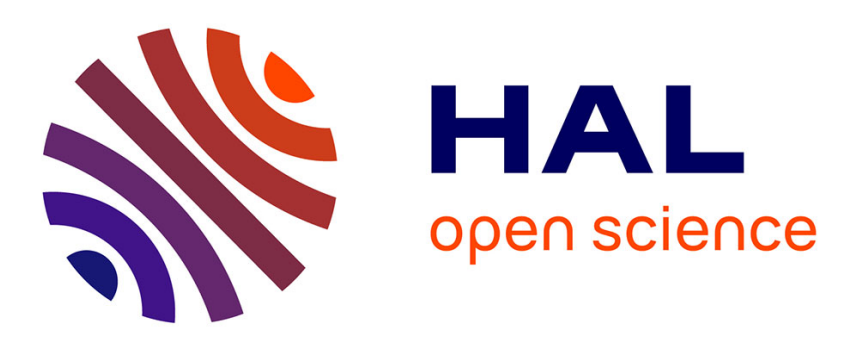

\title{
Quasi-Barabanov Semigroups and Finiteness of the L_2-Induced Gain for Switched Linear Control Systems: Case of Full-State Observation
}

Yacine Chitour, Paolo Mason, Mario Sigalotti

\section{- To cite this version:}

Yacine Chitour, Paolo Mason, Mario Sigalotti. Quasi-Barabanov Semigroups and Finiteness of the L_2-Induced Gain for Switched Linear Control Systems: Case of Full-State Observation. 54th IEEE Conference on Decision and Control (CDC), Dec 2015, Osaka, Japan. 10.1109/cdc.2015.7402985 . hal-01216017

\section{HAL Id: hal-01216017 \\ https://hal.inria.fr/hal-01216017}

Submitted on 11 Apr 2020

HAL is a multi-disciplinary open access archive for the deposit and dissemination of scientific research documents, whether they are published or not. The documents may come from teaching and research institutions in France or abroad, or from public or private research centers.
L'archive ouverte pluridisciplinaire HAL, est destinée au dépôt et à la diffusion de documents scientifiques de niveau recherche, publiés ou non, émanant des établissements d'enseignement et de recherche français ou étrangers, des laboratoires publics ou privés. 


\title{
Quasi-Barabanov semigroups and finiteness of the $L_{2}$-induced gain for switched linear control systems: case of full-state observation
}

\author{
Yacine Chitour, Paolo Mason, Mario Sigalotti
}

\begin{abstract}
Motivated by an open problem posed by J.P. Hespanha we extend the notion of Barabanov norm and extremal trajectory to general classes of switching signals. As a consequence we characterize the finiteness of the $L_{2}$-induced gain for a large set of switched linear control systems in case of full-state observation in terms of the sign of the generalized spectral radius associated with minimal realizations of the original switched system.
\end{abstract}

\section{INTRODUCTION}

Let $n, m, p$ be integers and $\tau$ a positive real number. Consider a switched linear control system

$\dot{x}=A_{\sigma} x+B_{\sigma} u, y=C_{\sigma} x+D_{\sigma} u,(x, u, y) \in \mathbb{R}^{n} \times \mathbb{R}^{m} \times \mathbb{R}^{p}$,

where $\sigma$ is in the class $\Sigma_{\tau}$ of piecewise constant signals with dwell time $\tau$ taking values in a fixed finite set $P$ of indices. Define the $L_{2}$-induced gain

$\gamma_{2}(\tau)=\sup \left\{\frac{\left\|y_{u}\right\|_{2}}{\|u\|_{2}} \mid u \in L_{2}\left((0, \infty), \mathbb{R}^{m}\right) \backslash\{0\}, \sigma \in \Sigma_{\tau}\right\}$

where $y_{u, \sigma}$ is the output corresponding to the trajectory of the system associated with $u$ and $\sigma$ starting at the origin at time $t=0$. In [5], Hespanha asked the following questions: (i) under which conditions is the function $\tau \mapsto \gamma_{2}(\tau)$ bounded? (ii) when $\gamma_{2}$ is not a bounded function over $(0, \infty)$, how to compute $\tau_{\min }$, the the infimum of the dwell-times $\tau>0$ for which $\gamma_{2}(\tau)$ is finite? (iii) how regular is $\gamma_{2}$ ?

In [6], Hespanha proved the surprising fact that, in general, $\lim _{\tau \rightarrow \infty} \gamma_{2}(\tau)>\max _{\sigma \in P} \gamma_{2}^{\sigma}$, where $\gamma_{2}^{\sigma}$ denotes the $L_{2}{ }^{-}$ induced gain of the time-invariant control system where $\sigma(\cdot) \equiv \sigma$. These results have been improved in [8], where $\gamma_{2}(\tau)$ is characterized in terms of a suitable switched Riccati equation, in the spirit of $H_{\infty}$ theory and worst-case switching laws. Similar questions have been considered in [7] for other classes of switching signals (average dwell-time, persistentdwell time, ...). In the latter work a crucial hypothesis for a class of switching signals has been put forward, namely, that of closure under concatenation.

In this paper we answer question (i) and characterize $\tau_{\min }$ from question (ii) in the case of full-state observation (i.e., $y=x$ ), both for classes $\Sigma_{\tau}$ of dwell-time switching signals as in [5] and for other classes $\Sigma$ of switching signals which are not closed under concatenation. The key tool in

Y. Chitour and P. Mason are with Laboratoire des Signaux et Systèmes (L2S, UMR CNRS 8506), CNRS - CentraleSupelec - Université Paris-Sud, 3, rue Joliot Curie, 91192, Gif-surYvette, France, yacine.chitour@lss.supelec.fr, paolo.mason@lss.supelec.fr.

M. Sigalotti is with INRIA Saclay, Team GECO \& CMAP, École Polytechnique, Palaiseau, France, mario.sigalotti@inria.fr our analysis is an extension of the notions of Barabanov norm and extremal trajectory [2] (see also [11]). The lack of closure under concatenation has been already addressed by Wirth in [12], where the author proves the continuity of the map $\tau \mapsto \rho(\tau)$, where $\rho(\tau)$ denotes the generalized spectral radius associated with the switched linear system $\dot{x}=A_{\sigma} x$ for $\sigma \in \Sigma_{\tau}$. The approach followed by Wirth is to build parameterized families of norms behaving as extremal norms on suitable subsets of $\Sigma_{\tau}$.

The construction adopted here to tackle the lack of closure under concatenation, although reminiscent of that of [12], follows a different path. We require that the class of switching signals $\Sigma$ admits at least one subset $\hat{\Sigma}$, large enough to encompass the asymptotic properties of the switched system $\dot{x}=A_{\sigma} x$ and well-behaved with respect to concatenation. The flows associated with $\hat{\Sigma}$ define a semigroup of matrices, which we call quasi-Barabanov semigroup. Its analysis allows us to point out the role played by special trajectories, which we call quasi-extremal and which describe the asymptotic worst-case behavior of the original switched system. Combining the notions of quasi-Barabanov semigroup and quasi-extremal trajectory with the characterization of controllability and observability for switched linear control systems given in [10] we prove, under full-state observation, that $\gamma_{2}$ is finite if and only if $\rho_{\min }<1$, where $\rho_{\min }$ is the generalized spectral radius associated with a minimal realization of the original switched control system (analogue to the situation in the unswitched case when one reduces the original problem to the controllable part of the system and then to the observable part of the latter system). In case of partial-state observation the situation is more complicated as we illustrate by means of an example: we construct a 3 dimensional switched linear control system with piecewise constant arbitrary switch which is controllable, observable, has finite $L_{2}$-induced gain, and whose uncontrolled dynamics have generalized spectral radius equal to one.

\section{PRELIMINARIES}

\section{A. Problem formulation}

The set of $n \times m$ matrices with real entries is denoted $M_{n, m}(\mathbb{R})$ and simply $M_{n}(\mathbb{R})$ if $n=m$. We use $\|\cdot\|$ to denote a norm on $\mathbb{R}^{n}$ and also the induced operator norm on $M_{n}(\mathbb{R})$. A subset $\mathcal{M}$ of $M_{n}(\mathbb{R})$ is said to be irreducible if an invariant subspace by the elements of $\mathcal{M}$ is either $\{0\}$ or $\mathbb{R}^{n}$. For every $s, t \geq 0$ and $A \in L_{\infty}\left([s, s+t], M_{n}(\mathbb{R})\right)$, denote by $\overrightarrow{\exp } \int_{s}^{s+t} A(\tau) d \tau \in M_{n}(\mathbb{R})$ the flow (or fundamental matrix) of $\dot{x}(\tau)=A(\tau) x(\tau)$ from time $s$ to time $s+t$. 
Consider switched linear control systems of the type

$$
\begin{aligned}
\dot{x}(t) & =A(t) x(t)+B(t) u(t), \\
y(t) & =C(t) x(t)+D(t) u(t),
\end{aligned}
$$

where $x \in \mathbb{R}^{n}, u \in \mathbb{R}^{m}, y \in \mathbb{R}^{p},(A(\cdot), B(\cdot), C(\cdot), D(\cdot))$ belongs to a class $\mathcal{T}$ of measurable switching laws taking values on a bounded set of triples of matrices $\mathcal{M}_{A} \times \mathcal{M}_{B} \times$ $\mathcal{M}_{C} \times \mathcal{M}_{D} \subset M_{n}(\mathbb{R}) \times M_{n, m}(\mathbb{R}) \times M_{p, n}(\mathbb{R}) \times M_{p, m}(\mathbb{R})$. We use $\pi_{A}$ and $\pi_{A \times B}$ to denote the projections on the first and the first two factors respectively and we set $\mathcal{T}_{A}=\pi_{A}(\mathcal{T})$ and $\mathcal{T}_{A \times B}=\pi_{A \times B}(\mathcal{T})$ respectively. Let $L_{2}$ be the Hilbert space of measurable functions $u(\cdot)$ defined on $[0, \infty)$ with finite $L_{2}$-norm, i.e., $\|u\|_{2}:=\left(\int_{0}^{\infty}\|u(t)\|^{2} d t\right)^{1 / 2}$ is finite.

For $u \in L_{2}$ and $(A(\cdot), B(\cdot), C(\cdot), D(\cdot)) \in \mathcal{T}$, let $x_{u}$ be the trajectory of Eq. (1) starting at the origin which is associated with $u$ and $(A(\cdot), B(\cdot), C(\cdot), D(\cdot))$ and $y_{u}$ the corresponding output. Finally, define the $L_{2}$-induced gain $\gamma_{2}$ associated with $\mathcal{T}$ by

$$
\gamma_{2}(\mathcal{T}):=\sup \left\{\begin{array}{ll}
\frac{\left\|y_{u}\right\|_{2}}{\|u\|_{2}} & \mid u \in L_{2} \backslash\{0\}, \\
& (A(\cdot), B(\cdot), C(\cdot), D(\cdot)) \in \mathcal{T}
\end{array}\right\} .
$$

We investigate conditions insuring the finiteness of $\gamma_{2}(\mathcal{T})$.

\section{B. Classes of switching functions}

We introduce in this section several standard classes of switching parameters which can meaningfully play the role of $\mathcal{T}_{A}$ in the framework described above. All classes are contained in $L_{\infty}([0, \infty), \mathcal{M})$, for some set $\mathcal{M} \subset M_{n}(\mathbb{R})$ (playing the role of $\mathcal{M}_{A}$ in the notations above).

- $\mathcal{S}_{\mathrm{arb}}(\mathcal{M})$ is the class of arbitrarily switching signals, i.e. $\mathcal{S}_{\text {arb }}(\mathcal{M})=L_{\infty}([0, \infty), \mathcal{M})$;

- $\mathcal{S}_{\mathrm{pc}}(\mathcal{M})$ is the class of piecewise constant signals;

- $\mathcal{S}_{\mathrm{d}, \tau}(\mathcal{M})$ is the class of piecewise constant signals with dwell-time $\tau>0$, i.e., such that the distance between two switching times is at least $\tau$ (notice that $\mathcal{S}_{\mathrm{pc}}(\mathcal{M})$ can be seen as $\mathcal{S}_{\mathrm{d}, 0}(\mathcal{M})$ );

- $\mathcal{S}_{\mathrm{BV}, T, \nu}(\mathcal{M})$ is the class of $(T, \nu)-B V$ signals, i.e., the signals whose restriction to every interval of length $T$ has total variation at most $\nu$, that is, $A(\cdot) \in$ $\mathcal{S}_{\mathrm{BV}, T, \nu}(\mathcal{M})$ if and only if

$$
\sup _{\substack{t \geq 0, k \in \mathbb{N} \\ t=t_{0} \leq t_{1} \leq \cdots \leq t_{k}=t+T}} \sum_{i=1}^{k}\left\|A\left(t_{i}\right)-A\left(t_{i-1}\right)\right\| \leq \nu .
$$

\section{ADAPTED NORMS FOR SWITCHED SYSTEMS WITH CONCATENABLE SUBFAMILIES}

We consider in this section a switched system $\dot{x}(t)=$ $A(t) x(t)$ where $A(\cdot)$ belongs to a class $\mathcal{S}$ of measurable switching laws taking values in a bounded set $\mathcal{M} \subset M_{n}(\mathbb{R})$.

From now on the family $\mathcal{S}$ is assumed to be shift-invariant, and we define the corresponding generalized spectral radius (see e.g. [12]) as

$$
\rho(\mathcal{S})=\limsup _{t \rightarrow+\infty} \sup _{A(\cdot) \in \mathcal{S}}\left\|\overrightarrow{\exp } \int_{0}^{t} A(\tau) d \tau\right\|^{1 / t} .
$$

Notice that, since $\mathscr{M}$ is bounded then $\rho(\mathcal{S})$ is finite.

Given two signals $A_{j}:\left[0, t_{j}\right] \rightarrow \mathcal{M}, j=1,2$, we denote by $A_{1} * A_{2}:\left[0, t_{1}+t_{2}\right] \rightarrow \mathcal{M}$ the concatenation of $A_{1}$ and $A_{2}$, i.e., the signal coinciding with $A_{1}(\cdot)$ on $\left[0, t_{1}\right]$ and with $A_{2}\left(\cdot-t_{1}\right)$ on $\left(t_{1}, t_{1}+t_{2}\right]$.

\section{A. Concatenable subfamilies}

Consider a set $\mathcal{F}=\cup_{t \geq 0} \mathcal{F}_{t}$ with $\mathcal{F}_{t} \subset L_{\infty}([0, t], \mathcal{M})$, $t \geq 0$. Define

$$
\hat{\mathcal{S}}=\left\{\begin{array}{c}
A_{1} * A_{2} * \cdots * A_{k} * \cdots \mid A_{k} \in \mathcal{F}_{t_{k}} \\
\text { for } k \in \mathbb{N}, \sum_{k \in \mathbb{N}} t_{k}=\infty
\end{array}\right\}
$$

and $\hat{\mathcal{R}}=\cup_{t \geq 0} \hat{\mathcal{R}}_{t}$, where, for every $t \geq 0$, $\hat{\mathcal{R}}_{t}=$ $\left\{\overrightarrow{\exp } \int_{0}^{t} A(\tau) d \tau \mid A(\cdot) \in \mathcal{F}_{t}\right\}$. Let, moreover,

$$
\mu(\mathcal{F})=\limsup _{t \rightarrow+\infty}\left(\sup \left\{\left\|R_{t}\right\|^{1 / t} \mid R_{t} \in \hat{\mathcal{R}}_{t}\right\}\right),
$$

with the convention that the quantity inside the parenthesis is equal to 0 if $\mathcal{F}_{t}$ is empty. Notice that $\mu(\mathcal{F}) \leq \rho(\hat{\mathcal{S}})$, but the converse is in general not guaranteed since the computation of $\rho(\hat{\mathcal{S}})$ takes into account all intermediate instants between two concatenation times, unlike the one of $\mu(\mathcal{F})$.

The results which follow are obtained taking as hypotheses some of the assumptions below, which list the main properties that one may require on $\mathcal{F}$ and $\hat{\mathcal{S}}$.

A1 (concatenability) $\mathcal{F}_{s} * \mathcal{F}_{t} \subseteq \mathcal{F}_{s+t}$ for every $s, t \geq 0$; A2 (irreducibility) $\hat{\mathcal{R}}$ is irreducible;

A3 (fatness) $\hat{\mathcal{S}} \subseteq \mathcal{S}$ and there exist two constants $C, \Delta \geq$ 0 and a compact subset $\mathcal{K}$ of GL $(n)$ such that for every $s, t \geq 0$ and $A(\cdot) \in \mathcal{S}$, there exist $K \in \mathcal{K}$, $\hat{t} \in[t, t+\Delta]$, and $Q \in \hat{\mathcal{R}}_{\hat{t}}$ such that

$$
\left\|\overrightarrow{\exp } \int_{s}^{s+t} A(\tau) d \tau K Q^{-1}\right\| \leq C .
$$

Moreover, if $A \in \hat{\mathcal{S}}$ and $s=0$, one can take $K=$ $\mathrm{I}_{n}$ in (4).

Remark 1: As consequence of the definition of $\hat{\mathcal{S}}$, if $A \in$ $\mathcal{F}$ and $B \in \hat{\mathcal{S}}$, then $A * B \in \hat{\mathcal{S}}$. Moreover, as a consequence of Assumption A1, one has that $\hat{\mathcal{R}}_{s} \hat{\mathcal{R}}_{t} \subset \hat{\mathcal{R}}_{s+t}$ for every $s, t \geq 0$. Hence $\hat{\mathcal{R}}$ is a semigroup and Assumption $\mathbf{A} 2$ above is equivalent to the following one

$\forall x \in \mathbb{R}^{n} \backslash\{0\}$, the linear span of $\hat{\mathcal{R}} x$ is equal to $\mathbb{R}^{n}$.

As in [11], one then says that $\hat{\mathcal{R}}$ is an irreducible semigroup.

Natural choices of $\mathcal{F}$ for the classes considered in the previous section are

- $\mathcal{F}_{\text {arb }}$ arbitrarily switching signals on finite intervals;

- $\mathcal{F}_{\text {pc }}$ piecewise constant signals on finite intervals;

- $\mathcal{F}_{\mathrm{d}, \tau}$ piecewise constant signals on finite intervals with dwell-time $\tau$ and such that the first and last subintervals on which the signal is constant have length at least $\tau$ (notice that $\left(\mathcal{F}_{\mathrm{d}, \tau}\right)_{t}=\emptyset$ for $t<\tau$ );

- $\mathcal{F}_{\mathrm{BV}, T, \nu}(T, \nu)$-BV signals on finite intervals $[0, t]$, $t \geq T$, starting and ending at some $\bar{M} \in \mathcal{M}$ fixed independently on the signal and constant on $[t-T, t]$. 
With these choices of $\mathcal{F}$ Assumption $\mathbf{A} 1$ is automatically satisfied. Assumption A3 is also easy to check. Indeed, every restriction $\left.A\right|_{[s, s+t]}$ of a signal in one of the classes $\mathcal{S}$ introduced in Section II-B can be extended to a signal $\left.A_{1} * A\right|_{[s, s+t]}(s+\cdot) * A_{2}$ in the corresponding class $\mathcal{F}$, with $A_{j}:\left[0, t_{j}\right] \rightarrow \mathcal{M}, j=1,2$, and $t_{1}, t_{2} \leq t_{*}$ for some $t_{*}$ uniform with respect to $A(\cdot) \in \mathcal{S}$, and $s, t \geq 0$.

As for Assumption A2, we have the following result.

Proposition 2: Let $\mathcal{M}$ be a bounded subset of $M_{n}(\mathbb{R})$, $\mathcal{S}$ a class of switching signals taking values in $\mathcal{M}$ and $\hat{\mathcal{S}}$ a subset of $\mathcal{S}$. Assume that $\mathcal{S}$ verifies in addition the following assumption.

A4 (piecewise-constant richness) there exists $\kappa \in \mathbb{N}$ such that, given any finite sequence $M_{0}, \ldots, M_{K} \in \mathcal{M}$, there exist $M_{-\kappa}, \ldots, M_{-1}, M_{K+1}, \ldots, M_{K+\kappa} \in$ $\mathcal{M}$ and $I_{-\kappa}, \ldots, I_{K+\kappa}$ open nonempty intervals in $(0, \infty)$ such that for every $t_{j} \in I_{j}, j=$ $-\kappa, \ldots, K+\kappa$, the concatenation $A_{-\kappa} * \cdots * A_{K+\kappa}$ is in $\mathcal{F}$, where $A_{j}:\left[0, t_{j}\right] \rightarrow \mathcal{M}$ is constantly equal to $M_{j}, j=-\kappa, \ldots, K+\kappa$.

If $\mathcal{M}$ is irreducible then $\mathbf{A 2}$ is satisfied.

Proof: See [4] for a proof.

\section{B. Quasi-Barabanov semigroups}

The main goal of this section consists in proving the result below.

Theorem 3: Given a class $\mathcal{S}$, assume that there exists a class $\hat{\mathcal{S}}$ such that Assumptions $\mathbf{A 1}-\mathbf{A} \mathbf{3}$ are satisfied. Then there exists a constant $C \geq 1$ such that for any $x_{0} \in \mathbb{R}^{n} \backslash\{0\}$ there exist a trajectory $x: t \mapsto \overrightarrow{\exp } \int_{0}^{t} A(\tau) d \tau x_{0}$ with $A$ belonging to the weak- $\star$ closure of $\hat{\mathcal{S}}$ such that for every $t \geq 0, \frac{1}{C} \rho(\mathcal{S})^{t}\left\|x_{0}\right\| \leq\|x(t)\| \leq C \rho(\mathcal{S})^{t}\left\|x_{0}\right\|$.

For that purpose, we introduce the following definitions. Let $\mathcal{M}, \mathcal{F}, \hat{\mathcal{S}}$, and $\hat{\mathcal{R}}$ as in the previous section.

Definition 4: 1) We say that $\hat{\mathcal{R}}$ is a quasi-extremal semigroup if there exists $C_{\mathrm{qe}}>0$ such that, for every $t \geq 0$ and $R \in \hat{\mathcal{R}}_{t}$, one has

$$
\|R\| \leq C_{\mathrm{qe}} \mu(\mathcal{F})^{t} .
$$

2) A quasi-extremal semigroup $\hat{\mathcal{R}}$ is said to be quasiBarabanov if there exists $C_{\mathrm{qb}}>0$ such that for every $x \in \mathbb{R}^{n}$ and $t \geq 0$ there exist $t^{\prime} \geq t$ and $R$ in $\hat{\mathcal{R}}_{t^{\prime}}$ such that

$$
\|R x\| \geq C_{\mathrm{qb}} \mu(\mathcal{F})^{t^{\prime}}\|x\| .
$$

3) A trajectory $x: t \mapsto \overrightarrow{\exp } \int_{0}^{t} A(\tau) d \tau x_{0}$ with $x_{0} \neq 0$ and $A(\cdot)$ belonging to the weak- $\star$ closure of $\hat{\mathcal{S}}$ is said to be quasi-extremal with constant $C_{\mathrm{qx}} \geq 1$ if for every $t \geq 0$

$$
\frac{1}{C_{\mathrm{qx}}} \mu(\mathcal{F})^{t}\left\|x_{0}\right\| \leq\|x(t)\| \leq C_{\mathrm{qx}} \mu(\mathcal{F})^{t}\left\|x_{0}\right\| .
$$

4) A quasi-extremal semigroup $\hat{\mathcal{R}}$ is said to be extremal if there exists a norm $w$ on $\mathbb{R}^{n}$ such that for every $t \geq 0$ and $R \in \hat{\mathcal{R}}_{t}$, one has $\|R\|_{w} \leq \mu(\mathcal{F})^{t}$. In this case we say that $w$ is extremal for $\hat{\mathcal{R}}$.
An extremal norm $w$ is said to be Barabanov for $\hat{\mathcal{R}}$ if the latter is a quasi-Barabanov semigroup and for every $t \geq 0$ and $C_{\mathrm{qb}}<1$, there exist $t^{\prime} \geq t$ and $R$ in $\hat{\mathcal{R}}_{t^{\prime}}$ such that $\|R\|_{w} \geq C_{\mathrm{qb}} \mu(\mathcal{F})^{t^{\prime}}$.

Given an extremal semigroup $\hat{\mathcal{R}}$, a trajectory $x: t \mapsto$ $\overrightarrow{\exp } \int_{0}^{t} A(\tau) d \tau x_{0}$ with $x_{0} \neq 0$ and $A$ in the weak- $\star$ closure of $\hat{\mathcal{S}}$ is said to be extremal for $\hat{\mathcal{R}}$ (and for the corresponding extremal norm $w$ ) if $w(x(t))=$ $\mu(\mathcal{F})^{t} w\left(x_{0}\right)$ for every $t \geq 0$.

Remark 5: If $\hat{\mathcal{R}}$ is quasi-extremal, then (6) holds true for every $t \geq 0$ and $R$ belonging to the closure of $\hat{\mathcal{R}}_{t}$. The same is true if $R=\lim _{k \rightarrow \infty} R_{k}$, where $R_{k} \in \hat{\mathcal{R}}_{t_{k}}$ and $\lim _{k \rightarrow \infty} t_{k}=t$.

Notice moreover that for every $A(\cdot)$ in the weak- $\star$ closure of $\mathcal{F}_{t}, \overrightarrow{\exp } \int_{0}^{t} A(\tau) d \tau$ is in the closure of $\hat{\mathcal{R}}_{t}$. This is a consequence of the continuity of the map $L_{\infty}([0, t], \mathcal{M}) \ni$ $A(\cdot) \mapsto \overrightarrow{\exp } \int_{0}^{t} A(\tau) d \tau \in M_{n}(\mathbb{R})$ with respect to the weak-ᄎ closure in $L_{\infty}([0, t], \mathcal{M})$ (see, for instance, [3, Proposition 21]).

Lemma 6: Let Assumptions $\mathbf{A 1}$ and $\mathbf{A 3}$ be satisfied. If $\hat{\mathcal{R}}$ is a quasi-Barabanov semigroup then there exists $C_{\mathrm{qx}} \geq 1$ such that any nonzero point of $\mathbb{R}^{n}$ is the initial condition of a quasi-extremal trajectory with constant $C_{\mathrm{qx}}$.

Proof: See [4] for the complete argument.

Set

$$
\begin{gathered}
\hat{\mathcal{R}}_{\infty}=\left\{R \mid \exists t_{k} \rightarrow \infty, R_{t_{k}} \in \hat{\mathcal{R}}_{t_{k}},\right. \\
\text { such that } \left.\mu(\mathcal{F})^{-t_{k}} R_{t_{k}} \rightarrow R\right\} .
\end{gathered}
$$

Following the same proof as in [11, Proposition 3.2], one can prove the proposition below.

Proposition 7: Let A1, A2 be satisfied and define $\hat{\mathcal{R}}$ and $\hat{\mathcal{R}}_{\infty}$ as above. Then

(i) $\quad \hat{\mathcal{R}}_{\infty}$ is compact and not reduced to $\{0\}$;

(ii) $\hat{\mathcal{R}}_{\infty}$ is a semigroup;

(iii) for every $t \geq 0, T \in \hat{\mathcal{R}}_{t}$ and $S \in \hat{\mathcal{R}}_{\infty}$, both $\mu(\mathcal{F})^{-t} T S$ and $\mu(\mathcal{F})^{-t} S T$ belong to $\hat{\mathcal{R}}_{\infty} ;$

(iv) $\hat{\mathcal{R}}_{\infty}$ is irreducible.

The following result can be proven as in [11, Lemma 3.4].

Proposition 8: Let A1, A2 be satisfied and define $\hat{\mathcal{S}}$ and $\hat{\mathcal{R}}_{\infty}$ as above. Let $\hat{v}: \mathbb{R}^{n} \rightarrow(0, \infty)$ be the function defined by

$$
\hat{v}(x)=\max _{R \in \hat{\mathcal{R}}_{\infty}}\|R x\|
$$

Then $\hat{v}$ is an extremal norm for $\hat{\mathcal{R}}$.

We have the following result.

Proposition 9: Let the class $\hat{\mathcal{S}}$ satisfy Assumptions A1A3. Then $\hat{\mathcal{R}}$ is an extremal and quasi-Barabanov semigroup. Proof: See [4] for the complete argument.

As a consequence of Lemma 6, we have the following.

Corollary 10: Let $\hat{\mathcal{S}}$ satisfy Assumptions A1-A3. Then there exists $C_{\mathrm{qx}} \geq 1$ such that $\hat{v}$ admits quasi-extremal trajectories starting at any nonzero point of $\mathbb{R}^{n}$.

Theorem 3 follows directly from Corollary 10 and the remark that $\mathbf{A 3}$ implies the equality $\rho(\mathcal{S})=\mu(\mathcal{F})$. 
IV. $L_{2}$-INDUCED GAIN FOR SWITCHED LINEAR CONTROL SYSTEMS

In this section we establish a first result on the finiteness of the $L_{2}$-induced gain associated with a switched linear control and a class $\mathcal{T}$ of signals in the case where the output map $y$ is equal to the full state $x$.

\section{A. Minimal realization for a switched linear control system}

Given a system of the type (1) associated with a class of signals $\mathcal{T}$, we first consider the corresponding objects when the dynamics is reduced to the reachable and observable sets, which are defined next. In what follows the matrix $D(\cdot)$ appearing in (1) is taken identically equal to zero and dropped from the notations. The general case can be reduced to this one as explained in [4] and [9].

We further assume that $\mathcal{T}_{A}=\pi_{A}(\mathcal{T})$ contains a subset $\hat{\mathcal{T}}_{A}$ satisfying Assumptions $\mathbf{A 1}$ and $\mathbf{A 3}$ with corresponding union of concatenable subfamilies denoted $\mathcal{F}$. Let $\hat{\mathcal{T}}$ the subset of $\mathcal{T}$ equal to $\pi_{A}^{-1}\left(\hat{\mathcal{T}}_{A}\right)$.

Definition 11: 1) A point $x \in \mathbb{R}^{n}$ is reachable in time $t \geq 0$ for the switched linear control (1) associated with $\mathcal{T}$ if there exist a switching law $(A(\cdot), B(\cdot)) \in \pi_{A \times B}(\hat{\mathcal{T}})$ and an input $u \in L_{2}$ such that $A_{\mid[0, t]} \in \mathcal{F}_{t}$ and the corresponding trajectory $x_{u}$ starting at 0 reaches $x$ in time $t$. The reachable set $\mathfrak{R}(\mathcal{T})$ associated with the switched linear control (1) is equal to the set of points $x \in \mathbb{R}^{n}$ for which there exists a time $t \geq 0$ so that $x \in \mathbb{R}^{n}$ is reachable in time $t$. The linear switched system is said to be controllable if $\Re(\mathcal{T})=\mathbb{R}^{n}$.

2) The observable set $\mathfrak{O}(\mathcal{T})$ associated with the switched linear control defined by (1) and $\mathcal{T}$ is equal to the set of points $x \in \mathbb{R}^{n}$ so that there exists a time $t \geq 0$ and a switching law $(A(\cdot), B(\cdot), C(\cdot)) \in \mathcal{T}$ such that the trajectory $x_{0}$ associated with the zero input and starting at $x$ gives rise to an ouput $y(\cdot)$ verifying $y(t) \neq 0$. The linear switched system is said to be observable if $\mathfrak{O}(\mathcal{T})=\mathbb{R}^{n}$.

Note that the reachable set $\mathfrak{R}(\mathcal{T})$ is usually defined as the union over $t \geq 0$ of the points $x \in \mathbb{R}^{n}$ reachable in time $t \geq 0$ by a trajectory starting at the origin and corresponding to a switching law $(A(\cdot), B(\cdot)) \in \pi_{A \times B}(\mathcal{T})$ and an input $u \in L_{2}$. Given a switched linear control system (1) and a class of switching signals $\mathcal{T}$, it is not clear that the corresponding reachable and observable sets $\mathfrak{R}(\mathcal{T})$ and $\mathfrak{O}(\mathcal{T})$ are linear subspaces. It has been shown in [10] that this is the case if $\mathcal{T}=\mathcal{S}_{\text {arb }}(\mathcal{M})$, where $\mathcal{M}=\left\{\left(A_{1}, B_{1}, C_{1}\right), \cdots,\left(A_{k}, B_{k}, C_{k}\right)\right\}$ with $k$ a positive integer. In addition, it is proved in the same reference that the state space admits direct sum decompositions in controllable (observable respectively) part for the linear switched system exactly as in the unswitched situation. More precisely, there exists a direct sum decomposition of the state space $\mathbb{R}^{n}=$ $\mathfrak{R}(\mathcal{T}) \oplus E$ and an invertible $n \times n$ matrix $P$ such that, if $r=\operatorname{dim} \mathfrak{R}(\mathcal{T})$ and $P^{-1} x=\left(x_{1}, x_{2}\right)^{T}$ with $x_{1} \in \mathfrak{R}(\mathcal{T})$, one has for $1 \leq j \leq k$

$P^{-1} A_{i}=\left(\begin{array}{cc}A_{i}^{c} & A_{i}^{2} \\ 0 & A_{i}^{3}\end{array}\right), P^{-1} B_{i}=\left(\begin{array}{c}B_{i}^{c} \\ 0\end{array}\right), C_{i} P=\left(C_{i}^{c} C_{i}^{2}\right)$, where the $A_{i}^{c}$ 's and the $B_{i}^{c}$ 's belong to $M_{r}(\mathbb{R})$ and $M_{r, m}(\mathbb{R})$ respectively. Moreover, the switched linear control system defined on $\mathbb{R}^{r}$ associated with $\mathcal{S}_{\text {arb }}\left(\mathcal{M}^{c}\right)$, where

$$
\mathcal{M}^{c}=\left\{\left(A_{1}^{c}, B_{1}^{c}, C_{1}^{c}\right), \cdots,\left(A_{k}^{c}, B_{k}^{c}, C_{k}^{c}\right)\right\},
$$

is completely controllable. We refer to $\mathcal{M}^{c}$ as the controllable part of $\mathcal{M}$. Note that one also gets that the corresponding output $y(\cdot)$ is equal to $y(\cdot)=C^{c}(\cdot) x_{1}(\cdot)$ and thus the original switched linear control system and the one reduced to the controllable space have same $L_{2}$-induced gains.

Similarly, there exists a direct sum decomposition of the state space $\mathbb{R}^{n}=\mathfrak{O}(\mathcal{T}) \oplus F$ and an invertible $n \times n$ matrix $Q$ such that, if $s=\operatorname{dim} \mathfrak{O}(\mathcal{T})$ and $Q^{-1} x=\left(x_{1}, x_{2}\right)^{T}$ with $x_{1} \in \mathfrak{O}(\mathcal{T})$, one has for $1 \leq j \leq k$

$$
\begin{gathered}
Q^{-1} A_{i} Q=\left(\begin{array}{cc}
A_{i}^{o} & 0 \\
A_{i}^{2} & A_{i}^{3}
\end{array}\right), Q^{-1} B_{i}=\left(\begin{array}{c}
B_{i}^{o} \\
B_{i}^{2}
\end{array}\right), \\
C_{i} Q=\left(\begin{array}{ll}
C_{i}^{o} & 0
\end{array}\right),
\end{gathered}
$$

where the $A_{i}^{o}$ 's and the $C_{i}^{o}$ 's belong to $M_{s}(\mathbb{R})$ and $M_{p, s}(\mathbb{R})$ respectively. Moreover, the switched linear control system defined on $\mathbb{R}^{s}$ associated with $\mathcal{S}_{\text {arb }}\left(\mathcal{M}^{o}\right)$, where

$$
\mathcal{M}^{o}=\left\{\left(A_{1}^{o}, B_{1}^{o}, C_{1}^{o}\right), \cdots,\left(A_{k}^{o}, B_{k}^{o}, C_{k}^{o}\right)\right\},
$$

is completely observable. We refer to $\mathcal{M}^{o}$ as the observable part of $\mathcal{M}$. Note that one also gets that the corresponding output $y(\cdot)$ is equal to $y(\cdot)=C^{o}(\cdot) x_{1}(\cdot)$ and thus the original switched linear control system and the one reduced to the controllable space have same $L_{2}$-induced gains.

From the above, one can proceed as follows. Consider a switched linear control system (1) associated with $\mathcal{S}_{\text {arb }}(\mathcal{M})$, where $\mathcal{M}=\left\{\left(A_{1}, B_{1}, C_{1}\right), \cdots,\left(A_{k}, B_{k}, C_{k}\right)\right\}$. First one reduces it to its controllable space $\mathfrak{R}\left(\mathcal{S}_{\text {arb }}(\mathcal{M})\right)$ and get a controllable switched linear control system associated with $\mathcal{S}_{\text {arb }}\left(\mathcal{M}^{c}\right)$ with same $L_{2}$-induced gain. Then, one reduces the latter system to its observable space $\mathfrak{O}\left(\mathcal{S}_{\text {arb }}\left(\mathcal{M}^{c}\right)\right)$ to finally obtain a switched linear control system associated with $\mathcal{S}_{\text {arb }}\left(\mathcal{M}^{r}\right)$ where $\mathcal{M}^{r}$ is the observable part of $\mathcal{M}^{c}$, which is controllable and observable and with $L_{2}$-induced gain equal to that of the original switched linear control system.

This motivates the following definition.

Definition 12: Consider the switched linear control system (1) associated with $\mathcal{S}_{\operatorname{arb}}(\mathcal{M})$, where $\mathcal{M}=$ $\left\{\left(A_{1}, B_{1}, C_{1}\right), \cdots,\left(A_{k}, B_{k}, C_{k}\right)\right\}$ with $A_{i} \in M_{n}(\mathbb{R}), B_{i} \in$ $M_{n, m}(\mathbb{R})$, and $C_{i} \in M_{p, n}(\mathbb{R}), 1 \leq i \leq k$. Then, there exist a nonnegative integer $n^{\prime} \leq n$ and a switched linear control system associated with $\mathcal{S}_{\text {arb }}\left(\mathcal{M}^{r}\right)$, where $\mathcal{M}^{r}=$ $\left\{\left(A_{1}^{r}, B_{1}^{r}, C_{1}^{r}\right), \cdots,\left(A_{k}^{r}, B_{k}^{r}, C_{k}^{r}\right)\right\}$ with $A_{i}^{r} \in M_{n^{\prime}}(\mathbb{R})$, $B_{i}^{r} \in M_{n^{\prime}, m}(\mathbb{R})$, and $C_{i} \in M_{p, n^{\prime}}(\mathbb{R}), 1 \leq i \leq k$, which is controllable and observable, and furthermore satisfies

$$
\gamma_{2}\left(\mathcal{S}_{\text {arb }}\left(\mathcal{M}^{r}\right)\right)=\gamma_{2}\left(\mathcal{S}_{\text {arb }}(\mathcal{M})\right) .
$$

We refer to such a switched linear control system as a minimal realization associated with (1) and $\mathcal{S}_{\text {arb }}(\mathcal{M})$.

Note that even though the integer $n^{\prime}$ is uniquely defined by the original switched linear control system, a minimal realization may not be unique since $\mathcal{M}^{r}$ depends on the choice 
of supplementary spaces to $\mathfrak{R}\left(\mathcal{S}_{\text {arb }}(\mathcal{M})\right)$ and $\mathfrak{O}\left(\mathcal{S}_{\text {arb }}\left(\mathcal{M}^{c}\right)\right)$ respectively. It can also be shown that the maximal Lyapunov exponent associated with the set $\left\{A_{i}^{r}, 1 \leq i \leq k\right\}$ does not depend on the specific minimal realization.

Finally, it must be recalled that [10] also provides a nice and explicit geometric description of $\mathfrak{R}\left(\mathcal{S}_{\text {arb }}(\mathcal{M})\right)$ and $\mathfrak{O}\left(\mathcal{S}_{\text {arb }}(\mathcal{M})\right)$ in terms of the data of the problem. Using that fact, we prove in [4], for all the classes $\mathcal{T}$ and corresponding concatenation of subfamilies of switching signals we consider in Section II-B verifying Assumption A4 (with switching signals taking values in some bounded set $\mathcal{N}$ ), that the reachable and observable sets (as in Definition 11) coincide with $\mathfrak{R}\left(\mathcal{S}_{\text {arb }}(\mathcal{N})\right)$ and $\mathfrak{O}\left(\mathcal{S}_{\text {arb }}(\mathcal{N})\right)$. As a consequence, we are able to define minimal realizations associated with a switched linear control system (1) and a class $\mathcal{T}$ (as in the previous paragraph) as in Definition 12 which corresponds to a class $\mathcal{T}^{r}$ of measurable switching laws taking values on a bounded set of triples of matrices $\mathcal{N}_{A}^{r} \times \mathcal{N}_{B}^{r} \times \mathcal{N}_{C}^{r} \subset$ $M_{n^{\prime}}(\mathbb{R}) \times M_{n^{\prime}, m}(\mathbb{R}) \times M_{p, n^{\prime}}(\mathbb{R})$, with $n^{\prime} \leq n$.

We then use $\mathcal{T}_{A}^{r}=\pi_{A}(\mathcal{T})$ and $\mathcal{T}_{A \times B}^{r}=\pi_{A \times B}(\mathcal{T})$ to denote the projections on the first factor and the two first factors respectively of $\mathcal{T}^{r}$.

\section{B. Case of full state observation}

We can now state the main result of this section.

Theorem 13: Let $\dot{x}(t)=A(t) x(t)+B(t) u(t)$ with output $y=x$ be a switched linear control system where $x \in \mathbb{R}^{n}$, $u \in \mathbb{R}^{p},(A(\cdot), B(\cdot))$ belongs to a class $\mathcal{T}$ of measurable switching laws taking values in a bounded set of matrices $\mathcal{M}_{A} \times \mathcal{M}_{B} \subset M_{n}(\mathbb{R}) \times M_{n \times p}(\mathbb{R})$. Consider a minimal realization $\dot{x}_{r}(t)=A_{r}(t) x(t)+B_{r}(t) u(t)$ with output $y_{r}=$ $x_{r}$ associated with a class $\mathcal{T}^{r}$ of switching systems, which is therefore controllable and so that $\gamma_{2}(\mathcal{T})=\gamma_{2}\left(\mathcal{T}^{r}\right)$. Assume moreover that:

(a) $\mathcal{T}_{A}^{r}$ contains a subset $\hat{\mathcal{T}}_{A}^{r}$ satisfying Assumptions A1, A3 and A4;

(b) the switched linear control system $\dot{x}_{r}(t)=$ $A_{r}(t) x(t)+B_{r}(t) u(t)$ associated with $\hat{\mathcal{T}}^{r}=$ $\pi_{A}^{-1}\left(\hat{\mathcal{T}}_{A}^{r}\right)$ is controllable.

Then $\gamma_{2}(\mathcal{T})$ is finite if and only if $\rho\left(\mathcal{T}_{A}^{r}\right)<1$.

Note that it is proved in [4] that $\rho\left(\mathcal{T}_{A}^{r}\right)$ does not depend on the specific choice of a minimal realization.

Proof: One can directly consider the minimal realization and then it is enough to show the theorem in the case where

(i) the linear switched system control system $\dot{x}(t)=$ $A(t) x(t)+B(t) u(t)$ with output $y=x$ associated with $\mathcal{T}$ is controllable;

(ii) the class $\mathcal{T}_{A}$ of switching signals contains a subset $\hat{\mathcal{T}}_{A}$ verifying Assumptions A1, A3 and A4;

(iii) the linear switched system $\dot{x}=A(t) x(t)+B(t) u(t)$ associated with $\hat{\mathcal{T}}:=\pi_{A}^{-1}\left(\hat{\mathcal{T}}_{A}\right)$ is controllable.

Assume first that $\rho\left(\mathcal{T}_{A}\right)<0$. Taking into account the definition (3) of $\lambda\left(\mathcal{T}_{A}\right)$ and the boundedness of $\mathcal{M}_{A}$, one gets the following exponential decay for the fundamental matrix of $A(\cdot)$ : there exists $C>0$ and $\lambda>0$ such that, for every $A(\cdot) \in \mathcal{T}_{A}$ and every $0 \leq s \leq t$, one has $\left\|\overrightarrow{\exp } \int_{s}^{t} A(\tau) d \tau\right\| \leq C e^{-\lambda(t-s)}$. As a consequence of the above and the boundedness of $\mathcal{M}_{B}$, one deduces that there exists $D>0$ such that, for every $u \in L_{2},(A(\cdot), B(\cdot)) \in \mathcal{T}$ and every $t \geq 0$, one has $\left\|x_{u}(t)\right\| \leq D \int_{0}^{t} e^{-\lambda(t-s)}\|u(s)\| d s$. If $\chi_{[0,+\infty)}(\cdot)$ denotes the characteristic function on $[0,+\infty)$, the integral function on the right-hand side of the above equation can be interpreted as the convolution $f_{1} * f_{2}$, where $f_{1}(t)=\chi_{[0,+\infty)}(t) e^{-\lambda t}$ and $f_{2}(t)=\chi_{[0,+\infty)}(t)\|u(t)\|$. That yields at once that $\left\|x_{u}\right\|_{2} \leq \frac{D}{\lambda}\|u\|_{2}$, hence the conclusion.

Assume now that $\rho\left(\mathcal{T}_{A}\right) \geq 1$. By a common linear change of coordinates we can put every matrix in $A \in \mathcal{M}_{A}$ into the upper triangular block form

$$
A=\left(\begin{array}{cccc}
A_{11} & A_{12} & \cdots & \\
0 & A_{22} & A_{23} & \cdots \\
\vdots & \ddots & \ddots & \ddots \\
0 & \cdots & 0 & A_{q q}
\end{array}\right)
$$

where each $A_{i i} \in M_{n_{i}-n_{i-1}}(\mathbb{R}), n_{i} \in \mathbb{N}$ and each $\mathcal{A}_{i}:=$ $\left\{A_{i i} \mid A \in \mathcal{M}_{A}\right\}$ is irreducible. Define the subsystems of $\mathcal{M}_{A}$ as the switched systems corresponding to the sets $\mathcal{A}_{i}$ and the class of switching signals $\mathcal{T}_{A, i}:=\left\{A_{i i}(\cdot) \mid A(\cdot) \in\right.$ $\left.\mathcal{T}_{A}\right\}$ for $i=1, \ldots, q$. Notice that the classes $\mathcal{T}_{A, i}$ and $\hat{\mathcal{T}}_{A, i}$ verify Assumptions A1-A4. Then every subsystem of $\mathcal{M}_{A}$ is irreducible and verifies $\rho\left(\mathcal{T}_{A, i}\right) \leq \rho\left(\mathcal{T}_{A}\right)$ for $1 \leq i \leq$ $q$, with equality holding for at least one index $i$. Since we only consider trajectories starting at zero, we can assume by eventually reducing the size of the system that $\rho\left(\mathcal{T}_{A, i}\right)<$ $\rho\left(\mathcal{T}_{A}\right)$ for $i=2, \ldots, q$ and $\rho\left(\mathcal{T}_{A, 1}\right)=\rho\left(\mathcal{T}_{A}\right)$.

Using Point (iii), there exists $\bar{x}:=x_{\bar{u}}(\bar{t})=$ $\left(\bar{x}_{1}, 0 \cdots, 0\right)^{T}$ with $\bar{x}_{1} \neq 0$ reachable in some time $\bar{t} \geq 0$, i.e., there exists a time $\bar{t} \geq 0$, a switching law $\left(A_{0}(\cdot), B_{0}(\cdot)\right)$ with $A_{0}(\cdot) \in \mathcal{F}_{\bar{t}}$ and a measurable function $\bar{u}(\cdot)$ defined on $[0, \bar{t}]$ so that $x_{\bar{u}}(\bar{t})=\bar{x}$. Let us identify $\bar{u}(\cdot)$ with its extension by zero on $[\bar{t}, \infty)$. The class $\hat{\mathcal{T}}_{A, 1}$ verifies the hypotheses of Theorem 3 and thus the corresponding semigroup $\hat{\mathcal{R}}_{1}$ is quasi-Barabanov and admits a quasi-extremal trajectory starting at $\bar{x}_{1}$ and corresponding to a signal $\hat{\mathcal{T}}_{A, 1}$ belonging to $\hat{S}_{A, 1}$. Note that if one extends such a trajectory to the full state space by adding zeros for the other coordinates, the resulting $\bar{x}(\cdot)$ is the trajectory of $\dot{x}=A(t) x(t)$ starting at $\bar{x}$ and corresponding to some signal $\bar{A}(\cdot)$ in the closure of $\hat{\mathcal{T}}_{A}$.

Let $\bar{E}(\cdot)$ be the signal defined as the concatenation of $A_{0}(\cdot)$ and $\bar{A}(\cdot)$ and let $A_{0,11}(\cdot)$ and $\bar{E}_{11}(\cdot)$ be the $(1,1)$ blocks corresponding to $A_{0}(\cdot)$ and $\bar{E}(\cdot)$ respectively. Let $\left(E^{l}\right)_{l \geq 0}$ (and then $\left.\left(E_{11}^{l}\right)_{l \geq 0}\right)$ be a sequence in $\hat{\mathcal{T}}_{A}\left(\hat{\mathcal{T}}_{A, 1}\right.$ respectively) weak- $\star$ converging to $\bar{A}$ ( $\bar{A}_{11}$ respectively). Therefore the sequence $\left(A_{0} * E^{l}\right)_{l \geq 0}\left(\left(A_{0,11} * E_{11}^{l}\right)_{l \geq 0}\right)$ is in $\hat{\mathcal{T}}_{A}\left(\hat{\mathcal{T}}_{A, 1}\right.$ respectively) and weak- $\star$ converges to $\bar{E}\left(\bar{E}_{11}\right.$ respectively). Moreover, for every positive time $t \geq 0$, the convergence of $s \mapsto \overrightarrow{\exp } \int_{0}^{s} E^{l}(\tau) d \tau$ to $s \mapsto \overrightarrow{\exp } \int_{0}^{s} \bar{E}(\tau) d \tau$ is uniform with respect to $s \in[0, t]$ and similarly for the corresponding $(1,1)$-blocks. One deduces that there exists a positive constant $C>0$ such that, for $l$ large enough and for every $s \in[0, t]$, one has $\left\|\overrightarrow{\exp } \int_{0}^{s} E^{l}(\tau) d \tau \bar{x}\right\| \geq$ $\frac{C}{2 C_{\mathrm{qx}}} \rho(\mathcal{T})^{s}\left\|\bar{x}_{1}\right\|$. Fix such an $l$ and define $\Gamma_{l}$ the trajectory 
of Eq.(1) starting at the origin and associated with $\bar{u}(\cdot)$ and $\left(A_{0} * E^{l}(\cdot), \bar{B}(\cdot)\right)$. The previous inequality exactly says that there exists a positive constant $C_{0}$, independent of $t \geq 0$ and $l$ such that, for every $s \in[\bar{t}, t+\bar{t}]$, one has $\left\|\Gamma_{l}(s)\right\| \geq$ $C_{0} \rho(\mathcal{T})^{s-t}$. That implies that there exists a positive constant $C_{1}$ independent of $t$ such that $\frac{\left\|\Gamma_{l}\right\|_{2}^{2}}{\|\bar{u}\|_{2}^{2}} \geq \frac{\int_{\bar{t}}^{t+\bar{t}}\left\|\Gamma_{l}(s)\right\|^{2} d s}{\|\bar{u}\|_{2}^{2}} \geq$ $C_{1} t$. Since all the trajectories $\Gamma_{l}$ are associated with the same input $\bar{u}(\cdot)$, the above inequality implies that $\gamma_{2}(\mathcal{T})$ defined in Eq.(2) is infinite.

Remark 14: It turns out that, for the classes of switching signals we consider in Section II-B, all the hypotheses of Theorem 13 are satisfied.

We can now answer some of the questions asked by Hespanha in [5].

Corollary 15: Let $\mathcal{M}$ be a bounded subset of $M_{n}(\mathbb{R}) \times$ $M_{n, p}(\mathbb{R})$ with $n, m$ positive integers and $\tau>0$. Consider the switched linear control system $\dot{x}=A(t) x(t)+B(t) u(t)$, $y=x$, where the switching signal $(A(\cdot), B(\cdot))$ belongs to the class $\mathcal{S}_{\mathrm{d}, \tau}(\mathcal{M})$ of piecewise constant signals with dwell-time $\tau>0$. Let $\gamma_{2}(\tau)$ be the $L_{2}$-induced gain associated with $\mathcal{S}_{\mathrm{d}, \tau}(\mathcal{M})$. Then, $\gamma_{2}(\tau)$ is finite if and only if $\rho\left(\mathcal{S}_{\mathrm{d}, \tau}(\mathcal{M})_{A}^{r}\right)<1$ and then

$$
\begin{aligned}
\tau_{\min } & =\inf \left\{\tau>0 \mid \gamma_{2}(\tau) \text { is finite }\right\} \\
& =\inf \left\{\tau>0 \mid \rho\left(\mathcal{S}_{\mathrm{d}, \tau}\left(\mathcal{M}_{A}^{r}\right)\right)<1\right\} .
\end{aligned}
$$

Note that there exists a reduced system associated with $\dot{x}=A(t) x(t)+B(t) u(t), y=x$ and $\mathcal{S}_{\mathrm{d}, \tau}(\mathcal{M})$ whose state space and switching signal values do not depend on $\tau$.

\section{Case of partial state observation}

Theorem 13 shows, in the case where the output $y$ is equal to the full state, that the necessary and sufficient condition for finiteness of the $L_{2}$-induced gain is exactly the same as in the unswitching framework. We exhibit below an example where this is not anymore true if the output $y$ is just a partial observation of the state $x$. More precisely, we define next a switched linear control system satisfying all the assumptions of Theorem 13 (with $\mathcal{T}_{A}=\mathcal{T}_{A}^{r}=\hat{\mathcal{T}}_{A}^{r}$ ), with the exception that the output is the projection on the third component of the system's state $\left(x_{1}, x_{2}, x_{3}\right)$, and whose $L_{2}$-induced gain is finite despite the fact that $\rho\left(\mathcal{T}_{A}\right)=1$.

Assume that $\mathcal{T}=\mathcal{S}_{\operatorname{arb}}(\mathcal{M})$ where $\mathcal{M}=$ $\left\{\left(A_{i}, b_{i}, c_{i}\right)\right\}_{i=1,2,3} \subset M_{3}(\mathbb{R}) \times \mathbb{R}^{3} \times \mathbb{R}^{3}$, with

$$
\begin{gathered}
A_{1}=\left(\begin{array}{ccc}
-1 & -\alpha & 0 \\
\alpha & -1 & 0 \\
0 & 0 & -1
\end{array}\right), A_{2}=\left(\begin{array}{ccc}
-1 & -\alpha & 0 \\
1 / \alpha & -1 & 0 \\
0 & 0 & -1
\end{array}\right), \\
A_{3}=\left(\begin{array}{ccc}
-4 & 0 & 1 \\
0 & -4 & 0 \\
1 & 0 & -1
\end{array}\right), \\
b_{1}=b_{2}=0, b_{3}=c_{1}=c_{2}=c_{3}=(0,0,1)^{T} .
\end{gathered}
$$

One easily checks that such a system is observable and controllable. We assume that the system associated with $\mathcal{M}^{\prime}=\left\{A_{1}, A_{2}\right\}$ is marginally stable (and reducible); this corresponds to a parameter value $\alpha \sim 4.5047$. Namely, in this case the system admits a closed (periodic) $\mathcal{C}^{1}$ trajectory
$\Gamma$ on the plane $x_{3}=0$ which can be completely determined analytically by using the results in [1]. In particular, up to rescaling, $\Gamma$ can be supposed to belong to the set $\left\{\left(x_{1}, x_{2}, 0\right) \subset \mathbb{R}^{3} \mid 1 \leq x_{1}^{2}+x_{2}^{2} \leq 3\right\}$, and the norm $v(x)=v\left(x_{1}, x_{2}\right)$ on the plane $x_{3}=0$ satisfying $v^{-1}(1)=\Gamma$ is a Barabanov norm for the restriction of $\mathcal{M}^{\prime}$ on that plane and satisfies $\|\nabla v(x)\| \leq \sqrt{3}$. Moreover, by homogeneity, $v(x)=\nabla v(x)^{T} \cdot\left(x_{1}, x_{2}, 0\right)^{T}$.

Let us consider the function $V(x)=\frac{1}{2}\left(v(x)^{2}+x_{3}^{2}\right)$. Then one shows that $0 \leq \liminf _{t \rightarrow \infty} V(x(t)) \leq-\frac{1}{4}\left\|x_{3}\right\|_{2}^{2}+$ $\|u\|_{2}\left\|x_{3}\right\|_{2}$, so that $\left\|x_{3}\right\|_{2} \leq 4\|u\|_{2}$, that is $\gamma_{2}(\mathcal{T}) \leq 4$. Notice that the computations above also show that $V(\cdot)$ is a weak Lyapunov function for $\mathcal{M}$, and in particular such a system is marginally stable, that is adding the matrix $A_{3}$ to the system given by $\mathcal{M}^{\prime}$ does not destabilize it.

Acknowledgements: This research was partially supported by the iCODE Institute, research project of the IDEX ParisSaclay, and by the Hadamard Mathematics LabEx (LMH) through the grant number ANR-11-LABX-0056-LMH in the "Programme des Investissements d'Avenir".

\section{REFERENCES}

[1] M. Balde, U. Boscain, and P. Mason. A note on stability conditions for planar switched systems. Internat. J. Control, 82(10):1882-1888, 2009.

[2] N. E. Barabanov. An absolute characteristic exponent of a class of linear nonstationary systems of differential equations. Sibirsk. Mat. Zh., 29(4):12-22, 222, 1988.

[3] A. Chaillet, Y. Chitour, A. Loria, and M. Sigalotti. Uniform stabilization for linear systems with persistency of excitation: the neutrally stable and the double integrator cases. Math. Control Signals Systems, 20(2):135-156, 2008.

[4] Y. Chitour, P. Mason, and M. Sigalotti. Quasi-Barabanov semigroups and finiteness of the $L_{2}$-induced gain for switched linear control systems. Preprint, 2015.

[5] J. P. Hespanha. $\mathcal{L}_{2}$-induced gains of switched linear systems. In V. D. Blondel and A. Megretski, editors, Unsolved Problems in Mathematical Systems \& Control Theory, pages 131-133. Princeton University Press, Princeton, NJ, 2003.

[6] J. P. Hespanha. Root-mean-square gains of switched linear systems. IEEE Trans. on Automat. Contr., 48(11), Nov. 2003.

[7] K. Hirata and J. P. Hespanha. $L_{2}$-induced gains of switched systems and classes of switching signals. In Proc. of the 49th Conf. on Decision and Contr., Dec. 2010.

[8] M. Margaliot and J. P. Hespanha. Root-mean-square gains of switched linear systems: a variational approach. Automatica J. IFAC, 44(9):2398-2402, 2008.

[9] M. Petreczky. Realization theory for linear switched systems: formal power series approach. Systems Control Lett., 56(9-10):588-595, 2007.

[10] Z. Sun, S. S. Ge, and T. H. Lee. Controllability and reachability criteria for switched linear systems. Automatica J. IFAC, 38(5):775786, 2002.

[11] F. Wirth. The generalized spectral radius and extremal norms. Linear Algebra Appl., 342:17-40, 2002.

[12] F. Wirth. A converse Lyapunov theorem for linear parameter-varying and linear switching systems. SIAM J. Control Optim., 44(1):210-239 (electronic), 2005. 